\title{
Macro-micro relationship in nanostructured functional composites
}

\author{
A. Zanotto ${ }^{1}$, A. Spinella ${ }^{1}$, G. Nasillo ${ }^{1}$, E.Caponetti $^{1,2}$, A. S. Luyt ${ }^{3 *}$ \\ ${ }^{1}$ Centro Grandi Apparecchiature, UninetLab, Via Marini 14, 90128 Palermo Italy \\ ${ }^{2}$ Dipartimento di Chimica "Stanislao Cannizzaro" Università degli studi di Palermo Viale delle Scienze 17, 90128 \\ Palermo, Italy \\ ${ }^{3}$ Department of Chemistry, University of the Free State, Private Bag X13, 9866 Phuthaditjhaba, South Africa
}

Received 11 October 2011; accepted in revised form 2 December 2011

\begin{abstract}
This paper examines the results of the characterization of two functional composites: Poly(methyl methacrylate) (PMMA)-Ce:YAG (yttrium aluminium garnet doped with cerium) and PMMA-cobalt hexacyanoferrate (CoHCF). The composites were prepared as possible emitters in the fields of lighting thermal sensors. The prepared composites were characterized using transmission electron microscopy (TEM), nuclear magnetic resonance (NMR) spectroscopy, thermogravimetric analysis (TGA), differential scanning calorimetry (DSC) and dynamic mechanical analysis (DMA) analyses to study the correlation between micro and macro characteristics. We found that the molecular interactions of the two different fillers with the matrix were localized in different sites of the polymer chains. Moreover, the composites showed an increased thermal strength and stiffness, in particular the PMMA-Ce:YAG composite.
\end{abstract}

Keywords: nanocomposites, PMMA, yttrium aluminium garnet doped with cerium (Ce:YAG), cobalt hexacyanoferrate (CoHCF), interfacial interaction

\section{Introduction}

The production of nanostructures, nanoparticles and, generally, nanostructured materials has become part of everyday scientific life, whether in the research or industrial laboratories. The embedding of nanofillers inside polymeric systems allows us to utilize the nano-features of (nanostructured) materials in addition to their functionality. Such combinations produce ductile materials possessing switchable functionalities, not only for basic research, but for the development of industrial applications.

The amount of filler used plays an important role in modifying the mechanical properties of polymers, creating new materials [1]. In functional composites the polymers allow the dispersed materials to better express their own qualities in three-dimensional space. The role of polymers is to guarantee a homo- geneous distribution for the nanofiller to exhibit a big surface area for optical applications. During composite formation it is important that the dispersed material stays in suspension, allowing continuity in the system.

The macroscopic characteristics of the composite often reflect its own microstructure. The properties of composites depend on the unique filler properties and on the morphology and interface features of the composite [2]. Such composites will exhibit interesting electric, optical and magnetic properties. The characteristics of these systems depend on the compatibility of the constituents and the sizes of their contact surfaces.

One of the challenges of preparing nanocomposite materials is to mix compatible and homogeneous nanofillers in a polymer matrix, guaranteeing or

\footnotetext{
${ }^{*}$ Corresponding author, e-mail: LuytAS@qwa.ufs.ac.za
}

(c) BME-PT 
improving the matrix performance. Poly(methyl methacrylate) (PMMA) is a commonly used thermoplastic matrix for fibres, sheet and particles. There have been several studies on PMMA-fibre composites prepared by in situ polymerization [3], solution mixing [4], or melt blending [5]. The last one is already an industrial process for fabricating carbon fibre-reinforced thermoplastic composites. A combination of solvent casting and melt mixing allowed to build composites with enhanced mechanical and electrical properties and exceptional fibrealignment [6]. Platelets and sheets also influence the composite's thermal and mechanical properties, in relation to the material's composition and dispersion state within the matrix [7].

Musbah et al. [8] have just discovered that nanopowders of phosphors $\mathrm{Y}_{2} \mathrm{O}_{3}\left(\mathrm{Eu}^{3+}\right)$ embedded in a PMMA matrix, prepared using a laboratory mixing molder, influence almost linearly the optical and dynamic mechanical properties of the nanocomposites. The intensity of the luminescence emission spectra and storage modulus, loss modulus and glass transition temperature $\left(T_{\mathrm{g}}\right)$ of the polymer composites increased with increasing content of the nanophosphor powder.

We prepared two nanostructured composites, cobalt hexacyanoferrate (CoHCF) nanoparticles in PMMA, and yttrium aluminium garnet doped with cerium (Ce:YAG) nanoparticles in PMMA. CoHCF nanoparticles show thermochromic behaviour, exhibiting a significant and non-reversible colour change around $100^{\circ} \mathrm{C}$. A new thermocromic optical sensor with memory could be developed from this new composite [9]. Ce:YAG, combined with GaN blue light emitting diodes (LED), is used for white light solid-state LED. In previous work it was described how the presence of filler (Ce:YAG) in the PMMA matrix can cause an organization of the structure into a lamellar shape around the particles [10]. This organization could influence the macro features of the composites.

The purpose of the present work was to study the possible relationships between macro- and microfeatures of PMMA/CoHCF and PMMA/Ce:YAG nanocomposites by means of transmission electron microscopy (TEM) and nuclear magnetic resonance (NMR) spectroscopy, as well as thermal and thermomechanical analyses. PMMA was chosen as matrix because of its transparency, ductility, recyclability and low price.

\section{Experimental \\ 2.1. Materials}

Potassium hexacyanoferrate(III) $\mathrm{K}_{3}\left[\mathrm{Fe}(\mathrm{CN})_{6}\right]$ (Sigma-Aldrich ACS reagent, 244023, $\geq 99.0 \%$, Schnelldorf, Germany), cobalt(II) chloride hexahydrate $\mathrm{CoCl}_{2} \cdot 6 \mathrm{H}_{2} \mathrm{O}$ (Fluka Purum, crystallized, $\geq 98.5 \%$, Schnelldorf, Germany) and 2,2-diethoxyacetophenone (Sigma-Aldrich, 227102, >95\%, Schnelldorf, Germany), and methyl methacrylate (MMA) (Sigma-Aldrich, M55909, $\leq 30$ ppm MEHQ as inhibitor, 99\%, Schnelldorf, Germany) were all used as received. CoHCF nanoparticles were obtained by the reaction of potassium hexacyanoferrate and cobalt chloride hexahydrate. $16.5 \mathrm{mg}$ of $\mathrm{K}_{3}\left[\mathrm{Fe}(\mathrm{CN})_{6}\right]$, dissolved into $5 \mathrm{~mL}$ of water $(0.01 \mathrm{M})$ were added to $5 \mathrm{~mL}$ of stirred water containing $17.8 \mathrm{mg}$ of $\mathrm{CoCl}_{2} \cdot 6 \mathrm{H}_{2} \mathrm{O}(0.015 \mathrm{M})$ at room temperature. This instantly created a dark red suspension. $\mathrm{Y}(\mathrm{NO} 3)_{3} \cdot 6 \mathrm{H}_{2} \mathrm{O}$ (Sigma-Aldrich, 237957, 99.8\%, Schnelldorf, Germany), $\mathrm{Al}\left(\mathrm{NO}_{3}\right)_{3} \cdot 9 \mathrm{H}_{2} \mathrm{O}$ (SigmaAldrich ACS reagent, 237973, $\geq 98 \%$, Schnelldorf, Germany), and $\mathrm{Ce}\left(\mathrm{NO}_{3}\right)_{3} \cdot 6 \mathrm{H}_{2} \mathrm{O}$ (Sigma-Aldrich, $392219,99.99 \%$, Schnelldorf, Germany) were the sources of $\mathrm{Y}(\mathrm{III}), \mathrm{Al}(\mathrm{III})$, and $\mathrm{Ce}(\mathrm{III})$ ions, respectively. The other reagents were ammonia solution (E. Merck, $25 \mathrm{v} / \mathrm{v} \%$ ) and 2-methacrylic acid (MAA) (Sigma-Aldrich, 155721, $250 \mathrm{ppm}$ MEHQ as inhibitor, 99\%, Schnelldorf, Germany).

Ce:YAG nanopowder was prepared by dissolving yttrium, aluminium, and cerium nitrates in deionized water. The hydroxides were precipitated by dropwise addition of $5 \mathrm{M}$ ammonia to the nitrates solution, under constant stirring, until a $\mathrm{pH}$ value of 8 . The gelatinous precipitate thus obtained was filtered and washed several times with water to remove the residual ammonia and nitrate ions. Then the precipitate was dried at $50^{\circ} \mathrm{C}$ for 24 hours. The mixed hydroxides were calcined at $900^{\circ} \mathrm{C}$ for two hours.

PMMA nanocomposite preparation: A $5 \mathrm{wt} \%$ suspension of filler nanopowder in MMA and MAA monomers (molar ratio 4:1) was formed. 2,2diethoxyacetophenone was added to start the photocuring process [3]. The dispersion was maintained under ultrasound and continuously irradiated 


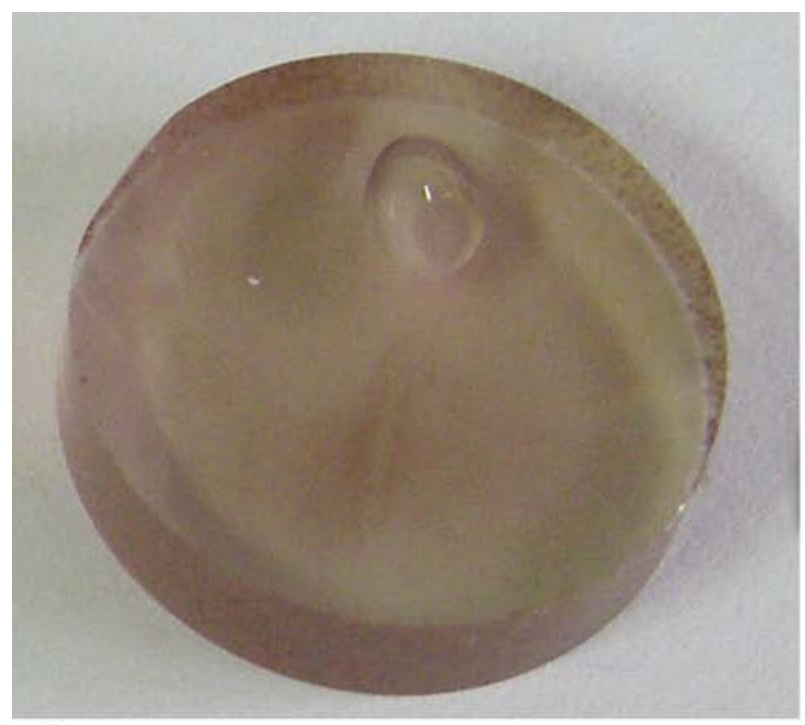

a)

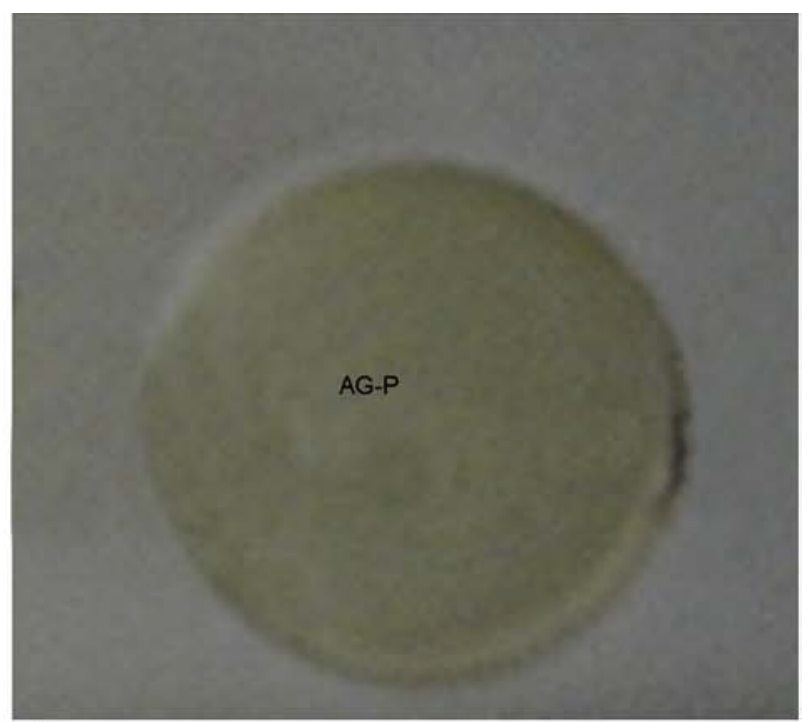

b)

Figure 1. Picture of CoHCF-PMMA (a) and Ce:YAG-PMMA (b) composites

with a $256 \mathrm{~nm}$ lamp until polymerization occurred. A transparent coloured solid product, composed of the PMMA polymeric matrix containing nanoparticles, was obtained. A portion was cut and lapped to obtain a disk having a diameter of $1 \mathrm{~cm}$. For the sake of comparison, pure PMMA was also prepared following the same procedure. The disks are shown in Figure 1.

\subsection{Methods}

\subsubsection{Transmission electron microscopy (TEM)}

TEM micrographs were recorded on a high-resolution transmission electron microscope (HR-TEM) JEOL JEM-2100 operating at $80 \mathrm{kV}$ accelerating voltage. Elemental analysis was carried out using an Oxford Instruments energy dispersive X-ray detector (EDS). The composite was cut into thin slices using a Leica EM-UC6 ultramicrotome. The $200 \mathrm{~nm}$ slices were deposited on a 200-mesh copper grid, which was carbon-coated to better disperse the high energy electron beam.

\subsubsection{Nuclear magnetic resonance (NMR) spectroscopy}

${ }^{13} \mathrm{C}$ cross-polarization magic-angle spinning nuclear magnetic resonance $\left({ }^{13} \mathrm{C}\left\{{ }^{1} \mathrm{H}\right\}\right.$ CP-MAS NMR $)$ spectra were obtained at room temperature using a Bruker Avance II $400 \mathrm{MHz}(9.4 T)$ spectrometer operating at $100.63 \mathrm{MHz}$ for the ${ }^{13} \mathrm{C}$ nucleus with a MAS rate of $13 \mathrm{kHz}$ for 1024 scans, a contact time of $1.5 \mu \mathrm{s}$, and a repetition delay of $2 \mathrm{~s}$. The optimization of the Hartmann-Hahn condition was obtained using an adamantine standard. All samples were placed in a $4 \mathrm{~mm}$ zirconia rotor with KEL-F caps using silica as a filler to avoid inhomogeneities inside the rotor.

The proton spin-lattice relaxation time in the rotating frame $T_{1 \rho}(\mathrm{H})$ was indirectly determined, with a variable spin-lock pulse sequence, by carbon nucleus observation using a 90- $\tau$ spin-lock pulse sequence prior to cross-polarization. Data acquisition was performed by ${ }^{1} \mathrm{H}$ decoupling with spin-lock pulse durations ranging from 0.1 to $7.5 \mathrm{~ms}$ and a contact time of $1.5 \mathrm{~ms}$.

\subsubsection{Differential scanning calorimetry (DSC)}

Differential scanning calorimetry (DSC) analyses were carried out in a Perkin Elmer DSC7 differential scanning calorimeter under flowing nitrogen atmosphere $\left(20 \mathrm{~mL} \cdot \mathrm{min}^{-1}\right)$. The instrument was calibrated using the onset temperatures of melting of indium and zinc standards, and the melting enthalpy of indium. Polymer samples (between 5 and $10 \mathrm{mg}$ ) were initially heated from 30 to $200^{\circ} \mathrm{C}$ at $10^{\circ} \mathrm{C} \cdot \mathrm{min}^{-1}$, held at that temperature for $1 \mathrm{~min}$ to eliminate thermal history effects, and then cooled to $30^{\circ} \mathrm{C}$ at $10^{\circ} \mathrm{C} \cdot \mathrm{min}^{-1}$. They were kept there for $1 \mathrm{~min}$, heated again to $200^{\circ} \mathrm{C}$ at $10^{\circ} \mathrm{C} \cdot \mathrm{min}^{-1}$, and cooled to $30^{\circ} \mathrm{C}$ at the same rate. 


\subsubsection{Thermogravimetric analysis (TGA)}

Thermogravimetric analyses (TGA) were carried out in a Perkin Elmer TGA7 thermogravimetric analyser. Polymer samples (between 5 and $10 \mathrm{mg}$ ) were heated from 30 to $850^{\circ} \mathrm{C}$ at $20^{\circ} \mathrm{C} \cdot \mathrm{min}^{-1}$ under flowing nitrogen $\left(20 \mathrm{~mL} \cdot \mathrm{min}^{-1}\right)$.

\subsubsection{Dynamic mechanical analysis (DMA)}

DMA analyses were conducted in a Perkin Elmer Diamond DMA using a dual cantilever in the torsion mode. The experiments were done at a frequency of $1 \mathrm{~Hz}$ and a temperature range of 25 to

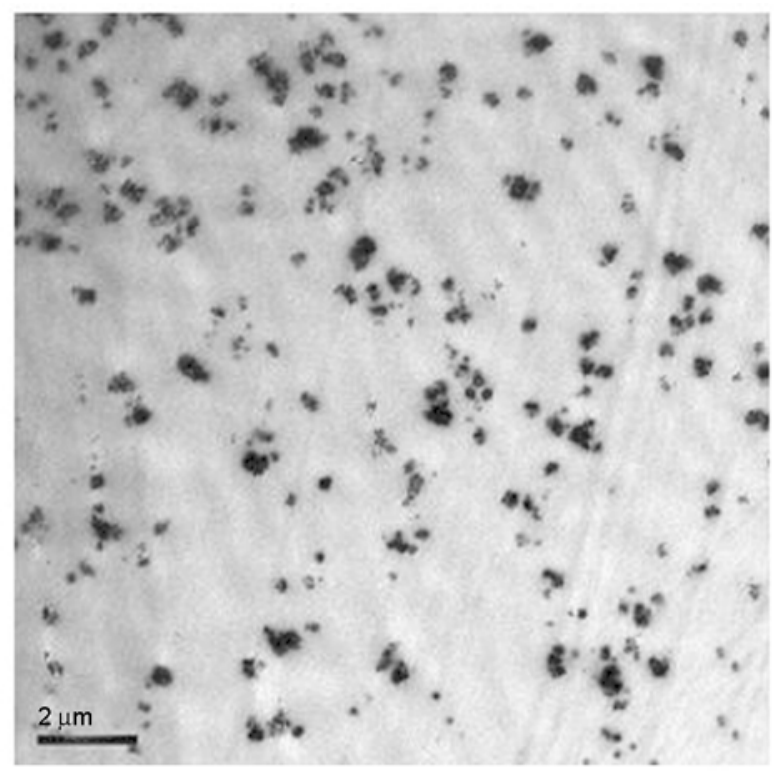

a)

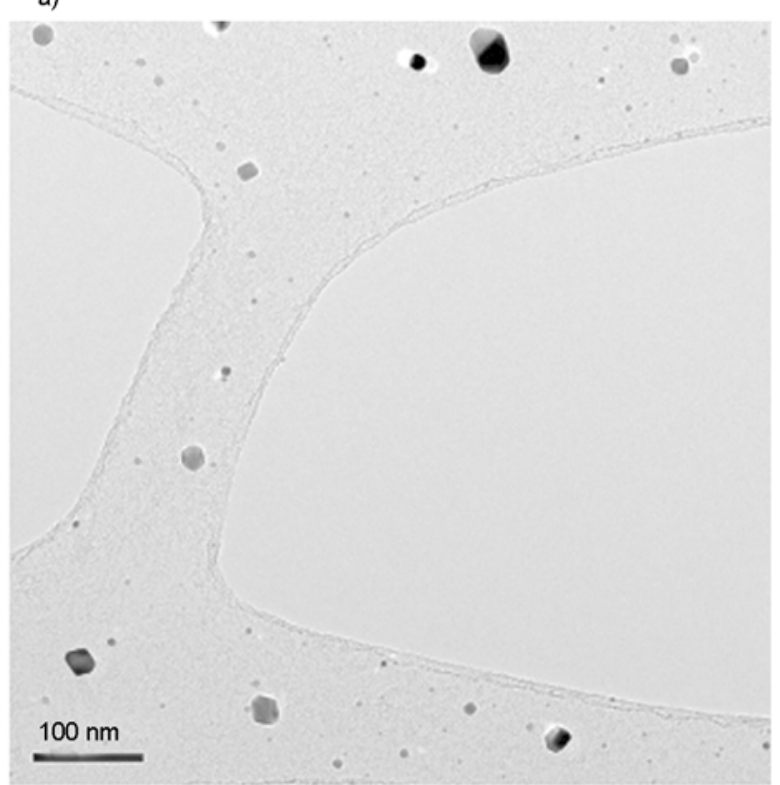

c) $170^{\circ} \mathrm{C}$. The sample dimensions were round disks of $10 \mathrm{~mm}$ diameter and $2.5 \mathrm{~mm}$ thick.

\section{Results and discussion}

A 4:1 polymeric blend of methylmethacrylate (MMA) and methacrylic acid (MAA) allowed the homogeneous dispersion of $\mathrm{CoHCF}$ nanoparticles and gave a dark red transparent composite, while Ce:YAG nanoparticles gave a yellow transparent composite (Figure 1).

Morphological and elemental characterization of a $200 \mathrm{~nm}$ thin slide of sample was carried out by

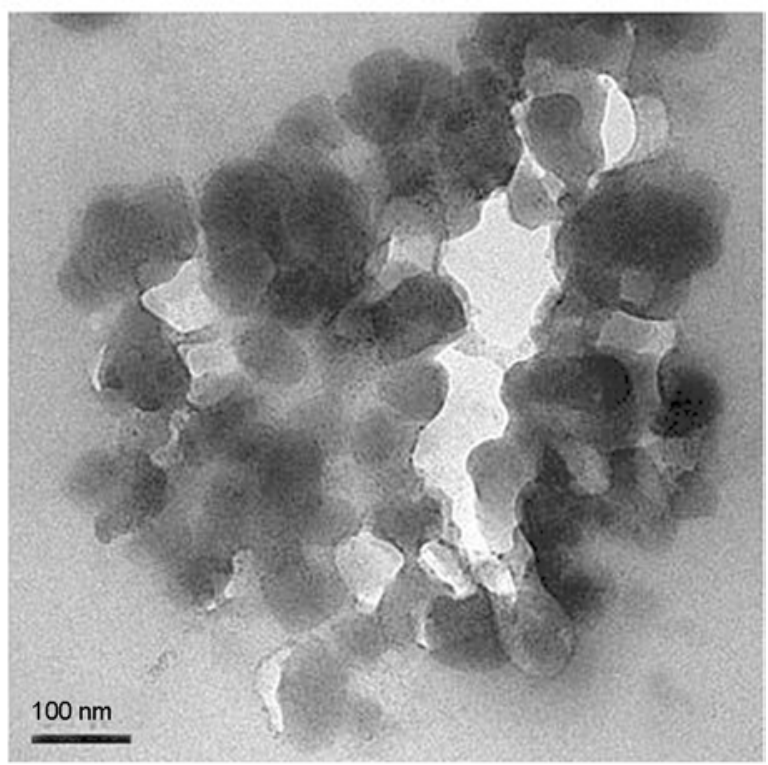

b)

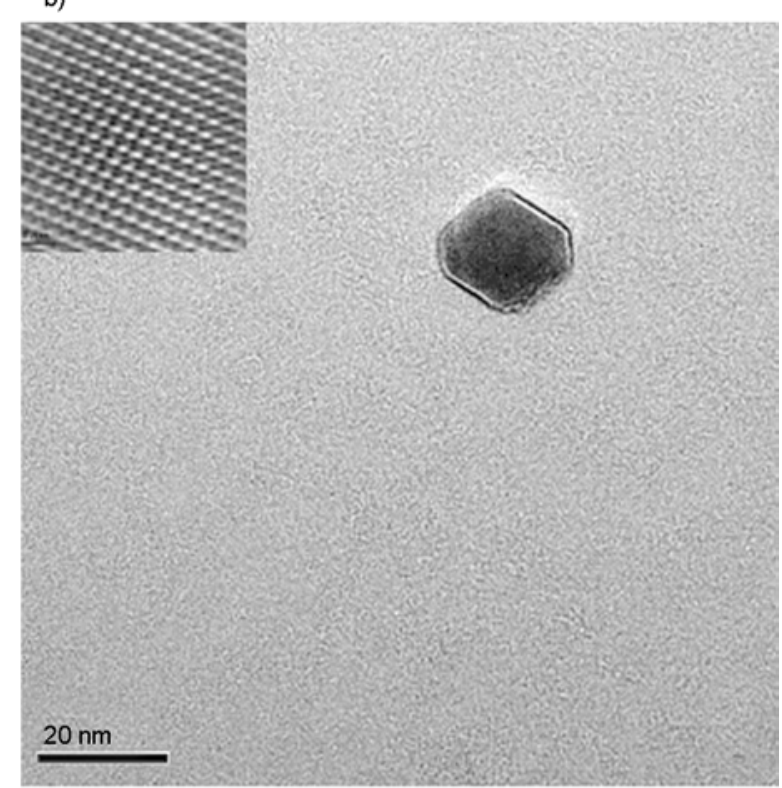

d)

Figure 2. TEM micrographs at different enlargements of PMMA-CoHCF (a and b) and PMMA-Ce:YAG (c and d) nanocomposites. In the insert is a proof of the crystallinity of Ce:YAG. 
TEM and EDS. The EDS investigations were necessary to confirm the composition of the samples. A representative example of a low enlargement micrograph is shown in Figure 2a, where a homogeneous distribution of CoHCF aggregates is observed. The CoHCF easily forms aggregates when the polar isopropanol-water solvent escapes during MMA polymerization. The reason is that in a concentrated environment the nanoparticles strongly attract each other and so the aggregates are more stable. The square structure of the CoHCF nanoparticles favours the square shape of the agglomerates. Figure $2 \mathrm{c}$ shows a homogeneous dispersion in the matrix of Ce:YAG nanoparticles with different sizes in the range 5$50 \mathrm{~nm}$. In this case the nanoparticles are dry when dispersed in the monomer, and therefore remain dispersed during the polymerization process. Unfortunately the Ce:YAG powder, during cutting operation, creates lacerations in the $100 \mathrm{~nm}$ polymer slice.

A single aggregate of $\mathrm{CoHCF}$ nanoparticles in the composite is shown in Figure $2 \mathrm{~b}$. The CoHCF aggregates are constituted by 15-25 nanoparticles randomly displaced with regular dimensions between 40 and $60 \mathrm{~nm}$. The nanoparticles have a quasi squared transversal section. Figure $2 \mathrm{~d}$ shows a single Ce:YAG nanoparticle with an average diameter smaller than $20 \mathrm{~nm}$. The insert in Figure $2 \mathrm{~d}$ confirms a highly crystalline matter.

The interaction between the filler and the matrix was studied by ${ }^{13} \mathrm{C}$-NMR CP-MAS spectroscopy and by acquiring spin-lattice relaxation times in the rotating reference system $\left(T_{1 \rho}\right)$. The ${ }^{13} \mathrm{C}-\mathrm{NMR}$ spectra of pure PMMA and the composites are reported in Figure 3. In all the spectra there are the character-

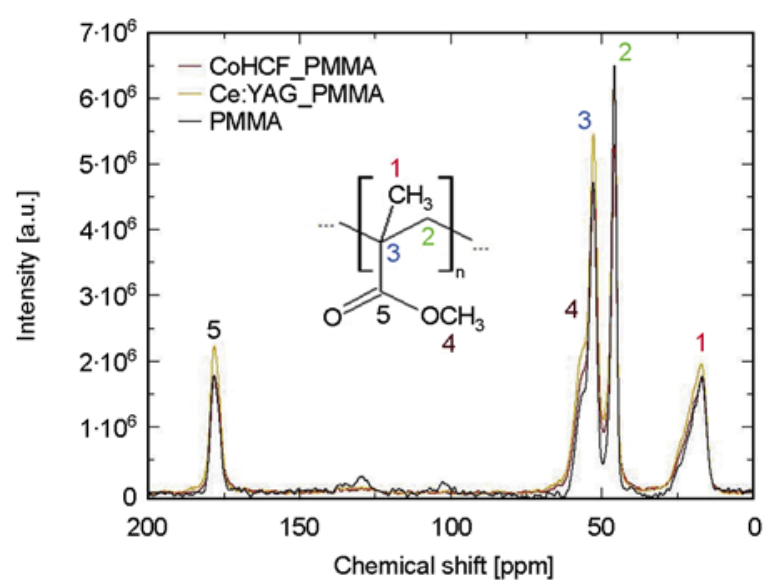

Figure 3. ${ }^{13} \mathrm{C},\left\{{ }^{1} \mathrm{H}\right\}$ CP-MAS NMR spectra of PMMA and its composites istic peaks of the PMMA matrix in agreement with literature [11]. When comparing the spectra, no new signals, changes in peak shapes or chemical shifts are observed, so the presence of nanofiller did not induce chemical modifications in the polymeric matrix.

The NMR data show some specific interactions. The spin-lattice $\left(T_{1 \rho}\right)$ relaxation times in the rotating frame are sensitive to molecular motions that occur in the $\mathrm{kHz}$ range [12]. These motions are typically associated with arrangements of the polymeric chains that develop from collective motions of a large number of monomeric units [13]. Long range cooperative motions are those motions that define a polymer's response to mechanical perturbations $[14,15]$. Moreover, $T_{1 \rho}$ in the $\mathrm{kHz}$ frequency range is inversely proportional to the spectral density of the motion.

The $T_{1 \rho}$ values of PMMA and the composites are reported in Table 1. The values in the table show an increase in the rigidity of both nanocomposites, probably due to immobilization of the polymer chains by means of filler-matrix interactions. The interactions are also locally different. The highest variation in the PMMA-CoHCF relaxation times is located in the quaternary (3) and methylenic (2) carbons on the backbone chain. An increase of $T_{1 \rho}(\mathrm{H})$ in PMMA-Ce:YAG occurs for peaks (4) and (5), the methoxylic and carbonylic groups. The interaction between the nanofiller and PMMA is principally localized around these nuclei.

The TGA curves of all three investigated samples are shown in Figure 4. It is clear that the presence of both $\mathrm{CoHCF}$ and Ce:YAG nanoparticles improves the thermal stability of PMMA. In the case of CoHCF the improvement is fairly insignificant, probably because the kinds of interaction between CoHCF and PMMA (as pointed out in the discussion of the NMR results) are not as strong as in the case of Ce:YAG, where there is an almost $50^{\circ} \mathrm{C}$ improvement in thermal stability. As shown in the discus-

Table 1. $T_{1 \rho}(\mathrm{H})$ values for all the peaks in the ${ }^{13} \mathrm{C}$ spectra of PMMA, PMMA-CoHCF and PMMA-Ce:YAG

\begin{tabular}{|c|r|c|c|c|}
\hline \multirow{2}{*}{ Peak } & \multirow{2}{*}{ ppm } & \multicolumn{3}{|c|}{ T $_{\mathbf{1} \boldsymbol{\rho}}(\mathbf{H})[\mathbf{m s}]$} \\
\cline { 3 - 5 } & & PMMA & CoHCF/PMMA & Ce:YAG-PMMA \\
\hline 1 & 16.4 & $6.6 \pm 0.2$ & $20.4 \pm 0.4$ & $9.0 \pm 0.4$ \\
\hline 2 & 45.5 & $7.0 \pm 0.2$ & $44.2 \pm 0.2$ & $12.8 \pm 0.2$ \\
\hline 3 & 52.7 & $7.6 \pm 0.3$ & $71.9 \pm 0.3$ & $13.6 \pm 0.3$ \\
\hline 4 & 55.1 & $7.2 \pm 0.2$ & $15.0 \pm 0.4$ & $15.0 \pm 0.4$ \\
\hline 5 & 178.1 & $6.4 \pm 0.4$ & $24.8 \pm 0.7$ & $24.8 \pm 0.7$ \\
\hline
\end{tabular}




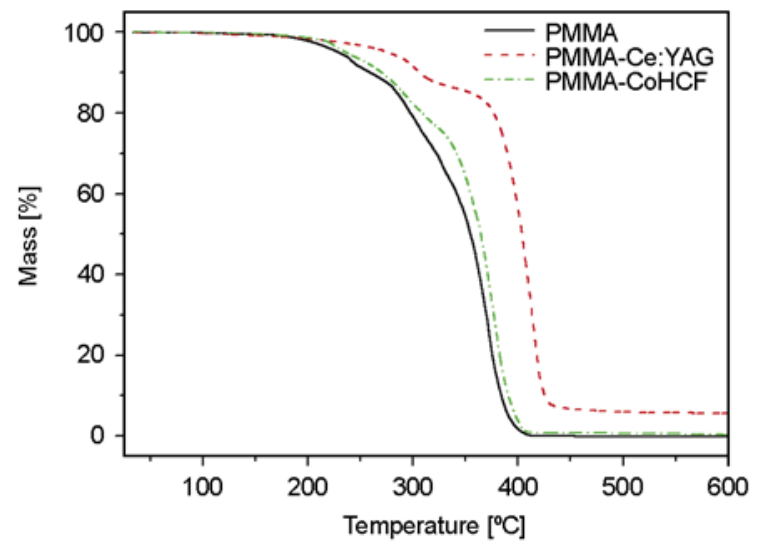

Figure 4. TGA curves of PMMA and its composites

sion above, there is a much better dispersion of Ce: YAG nanoparticles in PMMA, and a much stronger interaction between PMMA and Ce:YAG. This will not only immobilize the polymer chains but also the free radicals that take part in the degradation process. This stronger interaction is probably also the result of a local structured distribution of PMMA chains around the Ce:YAG nanoparticles [10].

The second heating DSC curve of PMMA-CoHCF (Figure 5) shows a shift in $T_{\mathrm{g}}$ to a higher temperature by about $6^{\circ} \mathrm{C}$. These results are reported in the Table 2. The higher glass transition temperature indicates the immobilization of the polymer chains because of their interaction with the nanoparticles. The DSC curve of PMMA-Ce:YAG shows an

Table 2. Glass transition temperatures for the different investigated samples

\begin{tabular}{|l|r|}
\hline Sample & $\left.\mathbf{T}_{\mathbf{g}}{ }^{\circ}{ }^{\mathbf{C}}\right]$ \\
\hline PMMA & 84.1 \\
\hline PMMA-CoHCF & 89.8 \\
\hline PMMA-Ce:YAG & 119.5 \\
\hline
\end{tabular}

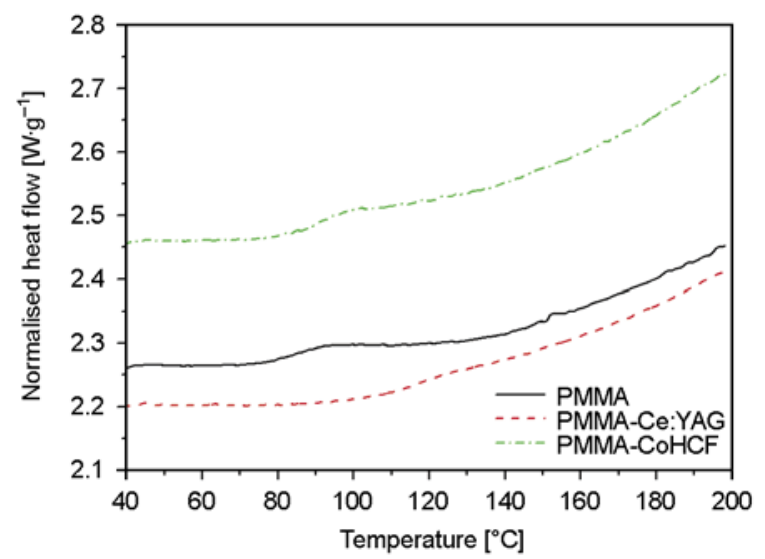

Figure 5. Second heating DSC curves of PMMA and its composites

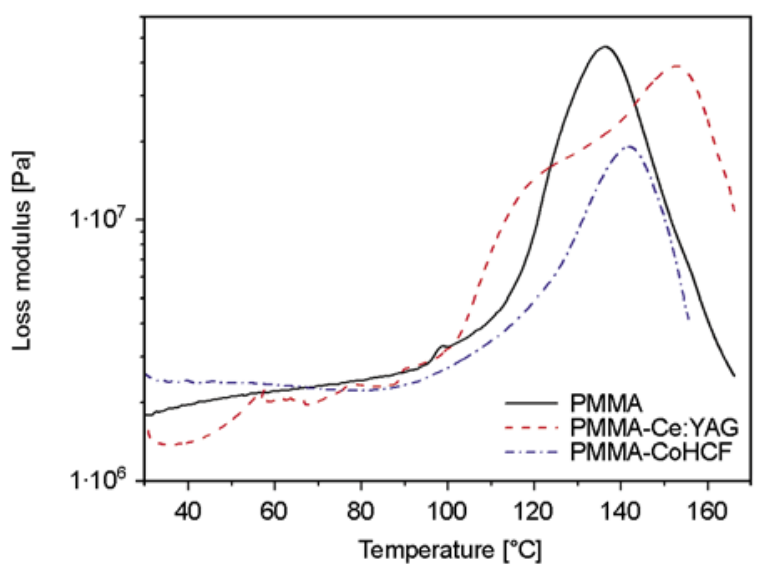

Figure 6. DMA loss modulus curves of PMMA and its composites

increase of $35^{\circ} \mathrm{C}$ in the glass transition temperature compared to that of the pure polymer. This transition is, however, not very well resolved. This shows that the interaction between the Ce:YAG nanoparticles and the PMMA chains, and the resultant immobilization of the polymer chains, was much stronger. The DMA loss modulus curves in Figure 6 confirm the DSC observations of the changes in the glass transition in the presence of the nanofillers. In this case, however, the $T_{\mathrm{g}}$ are significantly higher than those observed in the DSC curves, but such behaviour was observed before [16, 17], and there could be a number of reasons for this. However, the important thing is that the difference between the $T_{\mathrm{g}}$ values for PMMA and PMMA-CoHCF is $6^{\circ} \mathrm{C}$, as was also observed from the DSC curves. The difference between these values for PMMA and PMMA$\mathrm{Ce}: \mathrm{YAG}$ is, however, only $17^{\circ} \mathrm{C}$ from the DMA curves, but it is quite possible that the $T_{\mathrm{g}}$ value from the DSC curve of PMMA-Ce:YAG is not completely correct because of the badly resolved glass transition.

\section{Conclusions}

Both the investigated PMMA-Ce:YAG and PMMACoHCF composites show a homogeneous distribution of nanoparticles inside the polymer. The CoHCF is mostly agglomerated, while the Ce:YAG shows more dispersed nanoparticles. The presence of these nanofillers improved the thermal stability of PMMA for both CoHCF and Ce:YAG, and in particular PMMA-Ce:YAG shows a $50^{\circ} \mathrm{C}$ improvement in thermal stability. The $T_{\mathrm{g}}$ of both composites were higher than that of the matrix, with the largest 
increase observed for PMMA-Ce:YAG. The interactions of CoHCF with PMMA are localized around the quaternary and methylenic carbons on the backbone chain, and those for PMMA-Ce:YAG are around the methoxylic and carbonylic groups.

\section{Acknowledgements}

The National Research Foundation of South Africa and the University of the Free State are acknowledged for financial support of this project.

\section{References}

[1] Tjong S. C.: Structural and mechanical properties of polymer nanocomposites. Materials Science and Engineering R: Reports, 53, 73-197 (2006).

DOI: $10.1016 /$ j.mser.2006.06.001

[2] Hussain F., Hojjati M., Okamoto M., Gorga R. E.: Review article: Polymer-matrix nanocomposites, processing, manufacturing, and application: An overview. Journal of Composite Materials, 40, 1511-1575 (2006). DOI: $10.1177 / 0021998306067321$

[3] Jia Z., Wang Z., Xu C., Liang J., Wei B., Wu D., Zhu S.: Study on poly(methyl methacrylate)/carbon nanotube composites. Materials Science and Engineering A, 271, 395-400 (1999).

DOI: 10.1016/S0921-5093(99)00263-4

[4] Stéphan C., Nguyen T. P., de la Chapelle M. L., Lefrant S., Journet C., Bernier P.: Characterization of singlewalled carbon nanotubes-PMMA composites. Synthetic Metals, 108, 139-149 (2000). DOI: $10.1016 / \mathrm{S} 0379-6779(99) 00259-3$

[5] Jin Z., Pramoda K. P., Xu G., Goh S. H.: Dynamic mechanical behavior of melt-processed multi-walled carbon nanotube/poly(methyl methacrylate) composites. Chemical Physics Letters, 337, 43-47 (2001). DOI: 10.1016/S0009-2614(01)00186-5

[6] Haggenmueller R., Gommans H. H., Rinzler A. G., Fischer J. E., Winey K. I.: Aligned single-wall carbon nanotubes in composites by melt processing methods. Chemical Physics Letters, 330, 219-225 (2000). DOI: 10.1016/S0009-2614(00)01013-7

[7] Potts J. R., Dreyer D. R., Bielawski C. W., Ruoff R. S.: Graphene-based polymer nanocomposites. Polymer, 52, 5-25 (2011). DOI: $10.1016 /$ j.polymer.2010.11.042
[8] Musbah S. S., Radojević V. J., Borna N. V., Stojanović D. B., Dramićanin M. D., Marinković A. D., Aleksić R. R.: PMMA- $\mathrm{Y}_{2} \mathrm{O}_{3}\left(\mathrm{Eu}^{3+}\right)$ nanocomposites: Optical and mechanical properties. Journal of the Serbian Chemical Society, 76, 1153-1161 (2011).

DOI: $10.2298 / \mathrm{JSC} 100330094 \mathrm{M}$

[9] Zanotto A., Matassa R., Saladino M. L., Berrettoni M., Giorgetti M., Zamponi S., Caponetti E.: Cobalt hexacyanoferrate-poly(methyl methacrylate) composite: Synthesis and characterization. Materials Chemistry and Physics, 120, 118-122 (2010). DOI: 10.1016/j.matchemphys.2009.10.032

[10] Saladino M. L., Zanotto A., Martino D. C., Spinella A., Nasillo G., Caponetti E.: Ce:YAG nanoparticles embedded in a PMMA matrix: Preparation and characterization. Langmuir, 26, 13442-13449 (2010).

DOI: $10.1021 / 1 \mathrm{a} 9042809$

[11] Eijkelenboom A. P. A. M., Maas W. E. J. R., Veeman W. S., Werumeus Buning G. H., Vankan J. M. J.: Tripleresonance fluorine-19, proton, and carbon-13 CP-MAS NMR study of the influence of PMMA tacticity on the miscibility in PMMA/poly(vinylidene fluoride) (PVF2) blends. Macromolecules, 25, 4511-4518 (1992). DOI: $10.1021 / \mathrm{ma} 00044 \mathrm{a} 009$

[12] Edzes H. T., Veeman W. S.: ${ }^{13}$ C NMR spin-lattice relaxation in solid poly(methyl methacrylate). Polymer Bulletin, 5, 255-261 (1981). DOI: $10.1007 / \mathrm{BF} 00254711$

[13] Boyer R. F.: Dependence of mechanical properties on molecular motion in polymers. Polymer Engineering and Science, 8, 161-185 (1968). DOI: 10.1002/pen.760080302

[14] Farrar T. C., Becker E. D.: Pulse and fourier transform NMR. Academic Press, New York (1971).

[15] Campbell I. D., Dwek R. A.: Biological spectroscopy. The Benjamin Cummings Publishing Corporation, Menlo Park (1984).

[16] Yazdi M. H., Lee-Sullivan P.: Determination of dual glass transition temperatures of a PC/ABS blend using two TMA modes. Journal of Thermal Analysis and Calorimetry, 96, 7-14 (2009). DOI: $10.1007 / \mathrm{s} 10973-008-9831-3$

[17] Narine S. S., Kong X., Bouzidi L., Sporns P.: Physical properties of polyurethanes produced from polyols from seed oils: I. Elastomers. Journal of the American Oil Chemists' Society, 84, 55-63 (2007). DOI: $10.1007 / \mathrm{s} 11746-006-1006-4$ 